\title{
THIN FILMS INVESTIGATIONS BY MEANS OF SPIN-WAVE RESONANCE*
}

\author{
A.Z. MaKsYmowicz \\ Institute of Computer Science, Academy of Mining and Metallurgy \\ Al. Mickiewicza 30, 30-059 Kraków, Poland

\section{L.J. MAKSYMOWICZ} \\ Institute of Electronics, Academy of Mining and Metaliurgy \\ Al. Mickiewicza 30, 30-059 Kraków, Poland \\ AND J.S.S. WHITING \\ Department of Physics, University of York, York Y01 5DD, England
}

(Received March 21, 1991)

Magnetic resonance technique may successfully be applied to determine some basic parameters such as $g$-factor, magnetization $M_{s}$ or anisotropy energy constant $K_{u}$ in thin magnetic films. These parameters are obtained from a ferromagnetic resonance experiment when uniform precession of $M_{\mathrm{s}}$ takes place. From spin-wave resonance one may extract very valuable information on the exchange constant $A$ or the surface conditions characterized by the surface anisotropy energy (or pinning parameters $\rho$ ). In fact, it is only spin-wave resonance or similar techniques which allow for measurements of $A, \rho$ or the coupling constant $K_{c}$ between ferromagnetic sublayers in multilayered structure. The magnetic phase diagram, temperature dependence of the spin-waves stiffness constant, and the anisotropy energy constant may also be listed as less common examples of spin-wave resonance technique application for the investigation of thin films. This paper presents a theoretical approach to typical examples of experimental results and their interpretation from spin-wave resonance measurements.

PACS numbers: 76.50.+g, 76.30.-v, 73.90. $+\mathrm{f}$

*This work was supported by the Project CPBP 01.08 . 


\section{Introduction}

In this paper we intend to illustrate what information about the material constants may be obtained from a magnetic resonance experiment. This is a vast subject and within the scope of this article we would only provide some selected examples, and by no means a complete review of what has been done. Ferromagnetic Resonance (FMR) and Spin-Wave Resonance (SWR) may be useful for investigation of some properties of magnetic materials. We speak about FMR when a uniform precession of magnetization takes place. SWR occurs if a wave vector $k$ of the dynamic component of the magnetization differs from zero. One can distinguish the two cases experimentally for different resonance spectra observed. In FMR case only a single line is expected while in SWR we get a sequence of several lines of different intensities. It is the set of pinning parameters $\rho_{1}, \rho_{2}$ on both surfaces of a film which controls the spectra. FMR corresponds to the limit of vanishing $\rho^{\prime} s$. In the standard approach $\rho \propto K_{\mathbf{s}}$, the surface anisotropy constant, and so FMR is expected for samples with no surface anisotropy. SWR may be considered as a more general case of $\rho \neq 0$ and $k \neq 0$. Thus picking out samples with a single line only (or deliberately influencing surface conditions of samples, if we can) we may select thin films of FMR.

The saturation magnetization $M_{s}, g$-factor, and bulk anisotropy are examples of parameters which can be obtained from FMR. The surface anisotropy constant $K_{\mathrm{s}}$ and exchange constant $A$ are obtained from SWR measurements. The parameters are fitted from the predicted spectra and so theoretical models employed for interpretation of the experiment would also briefly be discussed in this paper.

The resonance takes place when the microwave frequency $\omega$ matches eigenfrequencies of normal magnetic excitations of the system. The frequencies of the normal modes may be altered by the value of external magnetic field $H$ or its direction $\Theta_{H}$ with respect to $z$-axis usually defined along the normal to the film. We observe the resonance as a rapid absorption of power by the sample placed in the microwave cavity when $H$ is scanned. The spectra are described by the intensity $I_{n}\left(\Theta_{H}\right)$ of $n$-th mode and its position $H_{n}\left(\Theta_{H}\right)$. We consider the limit of no energy dissipation when the linewidth $\Delta_{n}$ tends to zero. This means, for example, that we ignore eddy current losses assuming isolating films, or at least the thickness $L$ being small enough to neglect this current. The sample must be uniform and its dimensions in the $x$ - and $y$-directions must be much larger than $L$. We then may consider the film as infinite in $x-y$ plane. These and other approximations are sometimes well justified, in some cases they are just necessary to make the model mathematically treatable. In this paper we adopt all these simplifications, mainly to keep along the main line to illustrate how the SWR technique may be applied for material science. The next Section is devoted to the standard surface anisotropy model. Section 3 presents some experimental results of temperature dependence of $M_{\mathrm{s}}, A$ and the spin-wave stiffness constant $D$ measured by means of SWR.

So far we have discussed single films which are homogeneous in the volume of the film, apart from the very surface which is described by extra surface anisotropy terms. In real samples, however, we expect a distribution of some ma- 
terial constants, such as $M_{\mathrm{s}}$, either across the film plane (due to the geometry of the film growing technique) or along the normal to the film (diffusion processes, temperature gradient during deposition). These aspects are very important, but they will not be discussed here for they are not in the main scope of this paper. We only briefly mention multilayer structures which, in a sense, are also non-homogeneous. A multilayer film is a sequence of a few ferromagnetic layers separated by a non-magnetic medium. Recently, coupled films in the multilayered structure are of current interest for several reasons. The interaction between layers and specific properties of interfaces alter both static and dynamic behaviour of thin films. The interaction which couples the ferromagnetic sublayers in the multilayered film separated by a non-magnetic medium (or sometimes by another ferromagnetic substance) is of major concern and may be described in terms of the effective anisotropy field $H_{\text {eff }}$ or the coupling constant $K_{\mathrm{c}}$ evaluated from the interaction coupling between the ferromagnetic sublayers. Some experimental data on the multilayered structure are also presented in Sect. 3.

\section{Theoretical background}

We apply the classical equation of motion for spins in ferromagnetically ordered substances to get the dispersion relation between the resonance field $H$ applied at the angle $\Theta_{H}$ and the wave vector $k$. The dispersion relation reads [1]:

$$
\begin{aligned}
& \left(\frac{\omega}{\gamma}\right)^{2}=\left(\frac{1}{M_{\mathrm{s}} \sin ^{2} \theta} \frac{\partial^{2} E}{\partial \varphi^{2}}+\frac{2 A}{M_{\mathrm{s}}} k^{2}\right)\left(\frac{1}{M_{\mathrm{s}}} \frac{\partial^{2} E}{\partial \theta^{2}}+\frac{2 A}{M_{\mathrm{s}}} k^{2}\right) \\
& -\left(\frac{1}{M_{\mathrm{s}} \sin \theta} \frac{\partial^{2} E}{\partial \varphi \partial \theta}\right)^{2},
\end{aligned}
$$

where $\theta$ and $\varphi$ are the angles of magnetization $M_{\mathrm{s}}$ in the spherical coordinate system, $\nu=\omega / 2 \pi$ is the microwave frequency, $\gamma$ is the giromagnetic factor and $A$ - the exchange constant. The magnetic anisotropy energy $E(\varphi, \theta)$ depends on the direction of static magnetization $M_{\mathrm{s}}$ and consists of the energy of the magnetic field applied at the angle $\Theta_{H}$ in $y-z$ plane, the demagnetization energy and uniaxial anisotropy term $E_{\mathrm{u}}=K \sin ^{2} \theta$, where $K$ is the volume anisotropy energy constant. The magnetization angle $\theta$ can be obtained from the static equilibrium condition $\partial E / \partial \varphi=0$ and $\partial E / \partial \theta=0$, from which $\varphi=\pi / 2$ and

$$
2 H \sin \left(\theta-\Theta_{H}\right)-B_{\text {eff }} \sin 2 \theta=0,
$$

where $B_{\text {eff }}$ is effective $\left(4 \pi M_{\mathrm{s}}\right)$ given by

$$
B_{\mathrm{eff}}=4 \pi M_{\mathrm{s}}-\frac{2 K}{M_{\mathrm{s}}} \text {. }
$$

For the three energy terms assumed in $E(\varphi, \theta)$, the dispersion relation (1) becomes

$$
\left(\frac{\omega}{\gamma}\right)^{2}=\left(H \cos \left(\theta-\theta_{H}\right)-B_{\text {eff }} \cos 2 \theta+\frac{2 A}{M_{\mathrm{s}}} k^{2}\right)
$$




$$
\times\left(H \cos \left(\theta-\Theta_{H}\right)-B_{\text {eff }} \cos ^{2} \theta+\frac{2 A}{M_{s}} k^{2}\right) .
$$

We get for the perpendicular geometry (when $\theta=\Theta_{H}=0$ ) and for FMR (uniform mode, $k=0$ )

$$
\left(\frac{\omega}{\gamma}\right)_{\perp}=H_{\perp}-B_{\text {eff }}
$$

In the parallel case when $\theta=\Theta_{H}=90^{\circ}$ and for $k=0$ we have

$$
\left(\frac{\omega}{\gamma}\right)_{\|}^{2}=H_{\|}\left(H_{\|}+B_{\text {eff }}\right) \text {. }
$$

Equations $(5,6)$ may be used to determine the $g$-factor and $B_{\text {eff }}$ from FMR experiment,

$$
B_{\text {eff }}=H_{\perp}+\frac{1}{2} H_{\|}-\left[H_{\|}\left(H_{\perp}+\frac{5}{4} H_{\|}\right)\right]^{1 / 2},
$$

which is $4 \pi M_{\mathbf{s}}$ for the case of no uniaxial volume anisotropy when $K=0$, see Eq. (3). If $M_{\mathrm{s}}$ is known from independent measurcments, we can find the bulk anisotropy constant $K$.

The exchange constant $A$ may be found from the spin-wave resonance spectra in the perpendicular case:

$$
\left(\frac{\omega}{\gamma}\right)_{\perp}=H_{\perp}-B_{\text {eft }}+\frac{2 A}{M_{s}} k^{2}
$$

if the lines positions $\left(H_{\perp}^{n}\right)$ and corresponding wave vectors $k_{n}$ of the modes are known. The allowed values $k_{n}$ may be calculated from the surface inhomogeneity models [1, 2]. For example, in the limiting case of strongly pinned spins on both surfaces one gets

$$
k_{n}=n \pi / L,
$$

where $n=1,3,5, \ldots$ for the permissible in this case odd modes and $L$ is the film thickness. The dispersion law (8) predicts then a linear dependence of resonance fields $\left(H_{\perp}^{n}\right)$ on the square of the mode number $n^{2}$ and we may find the exchange constant $A$ from the slope of this linear dependence.

In general, finding the allowed values $k_{n}$ of the wave vector is a bit more complex. Formula (9) comes from the equation of motion when it is applied to the surface spins. Such spins experience different exchange energies (lack of some neighbours) and an extra torque due to the surface anisotropy $K_{\mathbf{s}}$ (or the pinning parameter $\left.\rho \propto K_{\mathrm{s}}\right)$. From the equation of motion one gets a set of $4 \times 4$ linear and uniform algebraic equations [1] for the dynamic magnetization $m(k)$. A non-zero solution exists only if the determinant vanishes. While scanning the applied field $H$ one obtains the resonance fields $H_{n}$ for any $\Theta_{H}$ and the dynamic magnetization 
$m$ is then given except for an arbitrary multiplication factor. The intensity is given by [1]:

$$
I_{n}=\frac{2}{L}\left[\int_{-L / 2}^{L / 2} \mathrm{~d} z m_{\varphi} / \mathrm{i}\right]^{2} / \int_{-L / 2}^{L / 2} \mathrm{~d} z\left[m_{\vartheta}^{2}+\left(m_{\varphi} / \mathrm{i}\right)^{2}\right]
$$

where $m_{\varphi}$ and $m_{\vartheta}$ are the spherical components of the dynamic microwave magnetization. $I_{n}$ is dimensionless and normalized to unity for the ferromagnetic (uniform) mode in the perpendicular resonance. It is seen that the arbitrary multiplication factor is irrelevant.

This completes the basic theoretical approach to SWR. For a given angle $\Theta_{H}$ of an applied magnetic field and assumed pinnings $\rho_{1}, \rho_{2}$ one gets the spectra: the mode labels $n$, the position of the lines $H_{n}$ and their intensities $I_{n}$. Fitting $H_{n}$ and $I_{n}$ to the experiment, we expect to find the surface pinnings $\rho_{1}, \rho_{2}$ from which some information may be extracted about the surface conditions.

It may happen that the zero of the determinant, leading to Eq. (9), does no longer exist for $\Theta_{I I}$ above some value $\Theta_{0}$. This means that we do not have that $n$-th mode any longer. We speak about the critical angle $\Theta_{0}$. There is another critical angle $\Theta_{\mathrm{c}}$ at which $I_{n}=0$. This is so when the nominator in Eq. (10) goes through zero and the mode reappears above $\Theta_{c}$. If the integral in the nominator is linear in $\Theta$ in vicinity of $\Theta_{c}$, then we may expect

$$
I_{n} \propto\left(\Theta-\Theta_{c}\right)^{2}
$$

Both the position and intensity of SWR are sensitive to the surface conditions (especially near the critical angles) characterized by the pinning parameters $\rho$. In fact, the pinning parameter is set up of two contributions coming from the surface anisotropy $K_{\mathrm{s}}$ and the variation of static magnetization $M_{\mathrm{s}}$ near the surface. In the circular precession approximation the pinning parameter is [3, 4]:

$$
\rho=\frac{K_{\mathrm{s}}}{A} \cos 20-\frac{\partial_{n} M_{\mathrm{s}}}{M_{\mathrm{s}}}
$$

where $\partial_{n} M_{\mathrm{s}}$ is the directional derivative of magnetization $M_{\mathrm{s}}$ near the surface. Due to different angular dependence we can, in principle, distinguish the two contributions experimentally.

The dependence of the microwave magnetization $m$ across the normal to the film shows that the modes are distributed in the volume of the sample, we call them volume modes. However, some of the solutions may correspond to negative $k^{2}$ which describes a surface mode since dynamic magnetization $m$ is then exponentially damped when moving off the film surface. Such modes are excited close to the surface of the thin film and, in general, may be provoked not only by negative pinning but also by the state of interfaces in multilayered structures, close-to-the-surface inhomogeneities of the magnetization and/or internal fields. The surface modes are always in the magnetic field range above the uniform mode resonance field $H_{\mathrm{u}}$. The intensity of surface modes is usually smaller than the corresponding intensity of the uniform mode $[5,6]$. The surface modes offer an 
alternative way of determination of surface anisotropy. In the limit of strong symmetrical negative pinning we get for the allowed wave vector of the surface mode $k=-\rho^{2}$ and then the dispersion relation (4) may be rewritten in the form more convenient for the interpretation of the experiment $[3,7]$ :

$$
\left[\left(H_{\mathrm{s}}-H_{\mathrm{u}}\right) \cos \left(\theta-\Theta_{H}\right)\right]^{1 / 2}=\left(\frac{2}{A M_{\mathrm{s}}}\right)^{1 / 2}\left|K_{\mathrm{s}}\right|\left(\cos 2 \theta-\frac{A}{K_{\mathrm{s}}} \frac{\partial_{n} M_{\mathrm{s}}}{M_{\mathrm{s}}}\right) .
$$

The left hand side of Eq. (13) is known: the resonance field $H_{\mathrm{s}}$ and direction of this field $\Theta_{H}$ from the direct measurements, the position $H_{\mathrm{u}}$ is calculated for FMR mode and the magnetization angle $\theta$ is given from the equilibrium condition (2). Thus plotting the left hand side of Eq. (13) against $\cos 2 \theta$ we expect a linear dependence with the slope proportional to $\left|K_{\mathrm{s}}\right|$ and the non-zero free term if there is non-vanishing $\partial_{n} M_{\mathrm{s}}$ contribution. The plot may be obtained for the angles up to a critical value at $\Theta_{H}=\Theta_{c}$ when the intensities of the surface mode vanish or the position of $H_{\mathrm{u}}$ coincides with the position of $H_{\mathrm{s}}, H_{\mathrm{s}}=H_{\mathrm{u}}$. Of course, the described method for determination of $K_{\mathrm{s}}$ is only effective when the basic parameters of the sample $M_{\mathrm{s}}, A$ or $g$-factor have been already known.

Recently there is a growing interest in multilayer films [8-13] that are a sequence of magnetic sublayers separated with non-magnetic ones. The magnetic layers are coupled through a non-magnetic medium and the problem of origin of the coupling, possible mechanisms and dependence of the coupling strength on the non-magnetic medium thickness are of extreme interest [11, 12]. In a simplified approach the multilayer structure may be simulated while adding the extra anisotropy energy term $[8,14]$, then the described mathematical treatment may be applied for the multilayered films. This is a rough yet satisfactory approximation for the purpose of this paper.

\section{Experiment}

As we mentioned earlier, in the standard approach $\partial_{n} M_{\mathbf{s}}=0$ and then $\vartheta_{\mathrm{c}}=45^{\circ}$ gives $\rho=0$, so that the intensity, apart from the uniform mode, vanishes. However, the observed values $[3,7,15-16]$ of the critical angles are different from $45^{\circ}$. This implies that either $\partial_{n} M_{\mathrm{s}} \neq 0$ in Eq. (12), as it was concluded in [3], or the circular precession approximation is not valid $[1,17]$. It comes out from calculations that the modes may disappear in pairs [17]. In the papers $[18,19]$ the authors scanned very carefully a rich SWR spectrum from the perpendicular to the parallel resonance. They observed as many as 11 lines. Moving off perpendicular orientation modes 11 and 10 vanished, then the pair 9 and 8 was missing followed by 7 and 6 until finally only 2 lines were left in the parallel case. The other type of the critical angle for which the mode reappears, see Eq. (11), is also reported in the experiment; Hoffman [16] observed a quadratic dependence of the intensities $I \propto\left(\theta-\theta_{c}\right)^{2}$ near the critical angle $\theta_{c}$ as it is expected.

The critical angles and SWR spectra near these angles are sensitive to the surface conditions described by the surface anisotropy constant $K_{\mathrm{s}}$ or gradient of the static magnetization $M_{\mathrm{s}}$ [20]. Maling use of the formula (13), not only the 
critical angles but also the anisotropy constants $K_{\mathrm{s}}$ in some GdCoMo films $[3,7]$ were found from the surface mode position near the critical angles. If we do not have the surface modes, we still may get some information on the values of $K_{\mathrm{s}}$ from the volume modes, usually by fitting to the experiment the observed ratio of the mode intensities.

The bulk or volume modes are more often used for finding the exchange constant $A$, the crucial parameter in magnetic materials. This is done from the slope of the predicted linear dependence, Eqs. $(8,9)$, of the resonance field in the perpendicular resonance on $n^{2}$, providing that $M_{\mathrm{s}}$ is known. In the first stage, we must confirm that the dependence is linear. Equation (9) holds only in the limit of large pinning parameter or for big $n$. So $A$ is found from the slope for higher modes, low-lying modes may deviate from the linear law. (Actually, this is due to the deviation that we may evaluate the surface constant $K_{\mathbf{s}}$.) For example, this is how $A$ was determined in some FeBSi films in the paper [10].

The just discussed linear dependence of the resonance field $H$ vs. $n^{2}$ for larger $n$ assumes that the sample is homogeneous. In amorphous substances, however, topological disorder must lead to some spatial fluctuations of the local structural or magnetic parameters such as the (volume) magnetic anisotropy, magnetization $M_{\mathrm{s}}$ or exchange constant $A$. These fluctuations alters the dispersion relation so that $H$ is no longer linear against $n^{2}$ even for big $n$ and the deviations are characteristic for each of the fluctuating parameters. This offers a tool to find out which fluctuations take place, their correlation lengths and amplitudes. An example of experimental study in that direction may be found in [21] for amorphous $\mathrm{FeB}$ and GdCoMo samples.

As it was discussed in the last Section, we may also determine the magnetization $M_{\mathrm{s}}$ from the magnetic resonance experiment. The temperature dependence $M_{\mathrm{s}}(T)$ in amorphous $\mathrm{FeB}$ and GdCoMo samples [21] and in magnetic semiconductors $\mathrm{CdCr}_{2} \mathrm{Se}_{4}[22,23]$ proved that the spin-wave theory $T^{3 / 2}$ law is nicely followed. The stiffness constant $D \propto T^{5 / 2}$ law was confirmed in [21].

In the paper [24], the effective coupling constant $K_{\mathrm{c}}$ between ferromagnetic sublayers was found as a function of the thickness $t$ of the non-magnetic medium and the number of non-magnetic layers in the multilayered structure of $\mathrm{FeBSi} / \mathrm{Si}$ and FeBSi/Pt. The surface energy anisotropy constant was also determined for FeBSi/Pt films. An additional anisotropy field of the effective coupling was found of easy-plane-type in the case of $\mathrm{Si}$ as the non-magnetic medium for $t$ greater than $1.5 \mathrm{~nm}$. For $\mathrm{Pt}$, the effective field exhibits an easy-axis anisotropy along the normal to the film and the surface anisotropy constant varies from sample to sample in the range from 0.2 to $0.6 \mathrm{erg} / \mathrm{cm}^{2}$. A weak $t$-dependence of the surface anisotropy field was also observed. The saturation magnetization $M_{\mathrm{s}}$, exchange constant $A$ and $g$-factor were determined from FMR and the spin-wave resonance experiment on single FeBSi films of the same composition as corresponding multilayer samples.

\section{Conclusions}

The ferromagnetic resonance and spin-wave resonance may be a useful tool for getting information about some material constants in thin films and about 
surface conditions. From FMR resonance fields in the perpendicular and parallel orientations one gets $g$-factor and magnetization $M_{\mathrm{s}}$, if the volume anisotropy constant is known. SWR provides some data on the exchange constant $A$ or the surface conditions characterized by the surface anisotropy $K_{\mathrm{s}}$ or static magnetization gradient at the surface $\partial_{n} M_{\mathrm{s}}$. The position of the resonance fields and especially the intensities of the modes are very sensitive to the surface conditions. In general, the surface anisotropy $K_{\mathrm{s}}$ and the gradient $\partial_{n} M_{\mathrm{s}}$ may equally contribute to the pinning and, therefore, they both should be taken into account. Calculations based on the circular precession approximation are acceptable only in the limit of small pinning or close to the perpendicular resonance. The temperature dependence of $M_{\mathrm{s}}$, spin-wave stiffness constant or magnetic phase diagram may be analyzed by the resonance technique. The dispersion relation for spin waves may be experimentally confirmed, for non-uniform samples we can sometimes extract some information on the character, amplitude and correlation length of the stochastic fluctuation of material parameters. In most cases the majority of lines, in a rich spectrum for the perpendicular case, vanishes for $\theta_{H}$ only up to only a few degrees. High accuracy of about 0.1 degree for sample orientation is required to experimentally investigate the transition and behaviour of modes intensities and resonance fields close to the critical angles.

In the multilayered structures we may measure the coupling constant $K_{\mathrm{c}}$ between ferromagnetic layers. When the position of the resonance peak for the multilayered structure is compared with the line position of a single film, the difference may be accounted for by the coupling field comprising contributions of different origin. For example, we may investigate this coupling as a function of temperature, thickness of the non-magnetic layer or against the number of the layers to find the dominant source for the coupling or a sort of competition of two or more mechanisms. If we choose different non-magnetic media, we may vary the conditions or stresses on the interfaces, or we may activate different sources of the coupling. $\mathrm{Pt}$ for example is close to ferromagnetism and so it should produce larger critical thickness above which the coupling is broken.

We may finally conclude that some essential magnetic properties may be studied by means of the resonance experiment.

\section{References}

[1] A.Z. Maksymowicz, Phys. Rev. B 33, 6045 (1986).

[2] II. Puszkarski, Prog. Surf. Sci. 0, 191 (1979).

[3] L.J. Maksymowicz, D. Sendorek, J. Magn. Magn. Mater. 37, 177 (1983).

[4] J. Spałek, W. Schmidt, Solid State Commun. 16, 193 (1975).

[5] G. Suran, R. Krishnan, Shiva Prasad, H. Jouve, R. Meyer, IEEE Trans. Magn. MAG-16, 1342 (1980).

[6] J.T. Yu, R.A. Turk, P.E. Wigen, Phys. Rev. B 11, 420 (1975).

[7] L.J. Maksymowicz, D. Sendorek, R. Żuberek, J. Magn. Magn. Mater. 46, 295 (1985).

[8] M. Pomerantz, J.C. Slonczewski, E. Spiller, J. Appl. Phys. 61, 3747 (1987). 
[9] P. Swiatek, F. Saurenbach, Y. Pang, P. Grŭnberg, W. Zinn, J. Appl. Phys. 61, 3753 (1987).

[10] L.J. Maksymowicz, A.Z. Maksymowicz, H. Jankowski, J. Magn. Magn. Mater. 73, 11 (1988).

[11] A. Laydi, I.O. Artman, B.O. Hall, R.A. Hoffman, C.L. Jensen, D.J. Chakrbanti, D.A. Saunders, 4-th JMMM Intermag Conf., Vancouver (Canada), 1988.

[12] M.J. Pechan, M.B. Salamon, I.K. Schuller, J. Appl. Phys. 55, 3678 (1988).

[13] A. Milewski, J. Samula, L.J. Maksymowicz, J. Wenda, H. Jankowski, A. Kulak, H. Lachowicz, 9th Internat. Conf. of Soft Magnetic Materials (Madrid 1989), to be published.

[14] M. Pomerantz, J.C. Slonczewski, E. Spiller, J. Magn. Magn. Mater. 54-57, 781 (1986).

[15] M. Okochi, H. Nose, J. Phys. Soc. Jpn 27, 312 (1969).

[16] F. Hoffman, Phys. Rev. B 4, 1604 (1971).

[17] A.Z. Maksymowicz, J Magn. Magn. Mater. 67, 14 (1987).

[18] D.F. Mitra, J.S.S. Whiting, J. Phys. F 8, 2401 (1978).

[19] J.S.S. Whiting, IEEE Trans. Magn. MAG-18, 709 (1982).

[20] A.Z. Maksymowicz, J.S.S. Whiting, Thin Solid Films 170, 191 (1989).

[21] L.J. Maksymowicz, D. Sendorek-Temple, R. Żuberek, J. Magn. Magn. Mater. 58, 303 (1986).

[22] M. Lubecka, W. Powroźnik, L.J. Maksymowicz, R. Żuberek, in: Surface Physics, Vol. III, Eds. A. Gieroszyński, J. Rutkowski, B. Sujak, Wyd. Uniwersytetu Łódzkiego, Łódź 1988, p. 246.

[23] M. Lubecka, W. Powroźnik, L.J. Maksymowicz, R. Żuberek, J. Phys. Colloq. 49, C8-867 (1988).

[24] L.J. Maksymowicz, H. Jankowski, A.Z. Maksymowicz, R. Żuberek, Thin Solid Films 191, 201 (1990). 